\title{
Tobit Censored NonParametric Regression Model in Plotting Employees
}

\author{
${ }^{1}$ Winda Rahma Ningrum, ${ }^{2}$ Helmi Agustina Hasibuan, ${ }^{3}$ Yulia Fitri \\ ${ }^{1,2,3}$ Department of Mathematics, University of North Sumatera, Indonesia \\ ${ }^{2}$ SMK Negeri 2 Binjai
}

\begin{abstract}
In this paper we consider identification and estimation of a censored nonparametric location scale model. We first show that in the case where the location function is strictly less than the (fixed) censoring point for all values in the support of the explanatory variables, then the location function is not identified anywhere. In contrast, if the location function is greater or equal to the censoring point with positive probability, then the location function is identified on the entire support, including the region where the location function is below the censoring point. In the latter case we propose a simple estimation procedure based on combining conditional quantile estimators for three distinct quantiles. The new estimator is shown to converge at the optimal nonparametric rate with a limiting normal distribution. A small scale simulation study indicates that the proposed estimation procedure performs well in finite samples. We also present an empirical application on plotting employees in a firm.
\end{abstract}

Keywords: Censored Regression, Tobit Estimator, Nonparametric Regression

\section{Introduction}

The nonparametric location-scale model is usually of the form:

$$
y_{i}=\mu\left(x_{i}\right)+\sigma\left(x_{i}\right) \epsilon_{i}
$$

where $\mathrm{x}_{\mathrm{i}}$ is an observed $\mathrm{d}$-dimensional random vector and $\epsilon_{i}$ is an unobserved random variable, distributed independently of $\mathrm{x}_{\mathrm{i}}$, and assumed to be centered around zero in some sense. The functions $\mu(\cdot)$ and $\sigma(\cdot)$ are unknown. In this paper, we consider extending the nonparametric location-scale model to accommodate censored data. The advantage of our nonparametric approach here is that economic theory rarely provides any guidance on functional forms in relationships between variables.

To allow for censoring, we work within the latent dependent variable framework, as is typically done for parametric and semiparametric models. We thus consider a model of the form:

$$
\begin{aligned}
y_{i}^{*} & =\mu\left(x_{i}\right)+\sigma\left(x_{i}\right) \epsilon_{i} \\
y_{i} & =\max \left(y_{i}^{*}, 0\right)
\end{aligned}
$$$$
y_{i}^{*}
$$

where is a latent dependent variable, which is only observed if it exceeds the fixed censoring point, which we assume without loss of marketingity is 0 . We consider identification and estimation of $\mu(x i)$ after imposing the location restriction that the median of $\epsilon_{\mathrm{i}}=0$. We emphasize that our results allow for identification of $\mu$ (xi) on the entire support of xi. This is in contrast to identifying and estimating $\mu(x i)$ only in the region where it exceeds the censoring point, which could be easily done by extending Powell's(1984) CLAD estimator to a nonparametric setting. One situation is when the data set is heavily censored. In this case, $\mu(x i)$ will be less than the
}

censoring point for a large portion of the support of $x i$, requiring estimation at these points necessary to draw meaningful inference regarding its shape.

Our approach is based on a structural relationship between the conditional median and upper quantiles which holds for observations where $\mu(x i) \geq 0$. This relationship can be used to motivate an estimator for $\mu(x i)$ in the region where it is negative. Our results are thus based on the condition

$$
P_{X}\left(x_{i}: \mu\left(x_{i}\right) \geq 0\right)>0
$$

where $\mathrm{PX}(\cdot)$ denotes the probability measure of the random variable $\mathrm{x}_{\mathrm{i}}$.

The paper is organized as follows. The next section explains the key identification condition, and motivates a way to estimate the function $\mu(\cdot)$ at each point in the support of $x_{i}$. Section 3 introduces the new estimation procedure and establishes the asymptotic properties of this estimator when the identification condition is satisfied. Section 4 considers an extension of the estimation procedure to estimate the distribution of the disturbance term. Section 5 explores the finite sample properties of the estimator through the results of a simulation study. Section 6 presents an empirical application STIFIN test, in which we estimate the survivor function in the region beyond the censoring point. Section 7 concludes by summarizing results.

\section{Estimation Procedure and Asymptotic Properties}

\subsection{Estimation Procedure}

In this section we consider estimation of the function $\mu(\cdot)$. Our procedure will be based on our identification results in the previous section, and involves nonparametric quantile regression at different quantiles and different points in the support of the regressors. Our asymptotic arguments are based on the local polynomial estimator for conditional 


\section{International Journal of Science and Research (IJSR) \\ ISSN (Online): 2319-7064}

Index Copernicus Value (2013): 6.14 | Impact Factor (2015): 6.391

quantile functions introduced in Chaudhuri(1991a,b). For expositional ease, we only describe this nonparametric estimator for a polynomial of degree 0 , and refer psycotesters to Chaudhuri(1991a,b), Chaudhuri et al.(1997), Chen and $\operatorname{Khan}(2000,2001)$, and $\operatorname{Khan}(2001)$ for the additional notation involved for polynomials of arbitrary degree.

First, we assume the regressor vector xi can be partitioned as $\left(\mathrm{xi}^{\mathrm{ds}}, \mathrm{x}^{\mathrm{c}}\right)$ where the $\mathrm{d}_{\mathrm{ds}}-$ dimensional vector $\mathrm{xi}^{\mathrm{ds}}$ is discretely distributed, and the $\mathrm{d}_{\mathrm{c}}$-dimensional vector $\mathrm{xi}^{\mathrm{c}}$ is continuously distributed.

We let $\operatorname{Cn}\left(\mathrm{x}_{\mathrm{i}}\right)$ denote the cell of observation $\mathrm{x}_{\mathrm{i}}$ and let $\mathrm{h}_{\mathrm{n}}$ denote the sequence of bandwidths which govern the size of the cell. For some observation $x_{j}, j \neq i$, we let $x_{j} \in C_{n}\left(x_{i}\right)$ denote that $\mathrm{x}_{\mathrm{j}}{ }^{(\mathrm{ds})}=\mathrm{x}_{\mathrm{i}}{ }^{(\mathrm{ds})}$ and $\mathrm{x}_{\mathrm{j}}{ }^{\odot}$ lies in the $\mathrm{d}_{\mathrm{c}}$-dimensional cube centered at $x_{i}^{c}$ with side length $2 h_{n}$.

Let $\mathrm{I}[\cdot]$ be an indicator function, taking the value 1 if its argument is true, and 0 otherwise. Our estimator of the conditional $\alpha^{\text {th }}$ quantile function at a point $\mathrm{x}_{\mathrm{i}}$ for any $\alpha \in(0$, 1 ) involves $\alpha$-quantile regression (see Koenker and Bassett (1978)) on observations which lie in the defined cells of $x_{i}$. Specifically, let $\theta$ minimize:

$$
\begin{gathered}
\sum_{j=1}^{n} I\left[x_{j} \in C_{n}\left(x_{i}\right)\right] \rho_{\alpha}\left(y_{j}-\theta\right) \\
\text { where } \rho_{\alpha}(\cdot) \equiv \alpha|\cdot|+(2 \alpha-1)(\cdot) I[\cdot<0] .
\end{gathered}
$$

Our estimation procedure will be based on a random sample of $n$ observations of the vector $\left(y_{i}, x_{i}\right)^{\prime}$ and involves applying the local polynomial estimator at three stages. Throughout our description, $\hat{\imath}$ will denote estimated values.

1) Local Constant Estimation of the Conditional Median Function. In the first stage, we estimate the conditional median at each point in the sample, using a polynomial of degree 0 . We will let $h_{1 n}$ denote the bandwidth sequence used in this stage. Following the terminology of Fan(1992), we refer to this as a local constant estimator, and denote the estimated values by q0.5(xi). Recalling that our identification result is based on observations for which the median function is positive, we assigns weights to these estimated values using a weighting function, denoted by $\mathrm{w}(\cdot)$. Essentially, w( $(\cdot)$ assigns 0 weight to observations in the sample for which the estimated value of the median function is 0 , and assigns positive weight for estimated values which are positive.

2) Weighted Average Estimation of the Disturbance Quantiles In the second stage, the unknown quantiles $c_{\alpha 1}$, $\mathrm{c}_{\alpha 2}$ are estimated (up to the scalar constant _c) by a weighted average of local polynomial estimators of the quantile functions for the higher quantiles $\alpha 1, \alpha 2$. In this stage, we use a polynomial of degree $\mathrm{k}$, and denote the second stage bandwidth sequence by $h_{2 n}$.
We let $\wedge 1, \hat{c} 2$ denote the estimators of the unknown constants $\frac{c_{\alpha_{1}}}{\Delta c}, \frac{c_{\alpha_{2}}}{\Delta c}$, and define them

$$
\hat{c}_{1}=\frac{\frac{1}{n} \sum_{i=1}^{n} \tau\left(x_{i}\right) w\left(\hat{q}_{0.5}\left(x_{i}\right)\right) \cdot \frac{\left(\hat{q}_{\alpha_{1}}\left(x_{i}\right)-\hat{q}_{0.5}^{(p)}\left(x_{i}\right)\right)}{\left(\hat{q}_{\alpha_{2}}\left(x_{i}\right)-\hat{q}_{\alpha_{1}}\left(x_{i}\right)\right)}}{\frac{1}{n} \sum_{i=1}^{n} \tau\left(x_{i}\right) w\left(\hat{q}_{0.5}\left(x_{i}\right)\right)}
$$

$$
\hat{c}_{2}=\frac{\frac{1}{n} \sum_{i=1}^{n} \tau\left(x_{i}\right) w\left(\hat{q}_{0.5}\left(x_{i}\right)\right) \cdot \frac{\left(\hat{q}_{\alpha_{2}}\left(x_{i}\right)-\hat{q}_{0.5}^{(p)}\left(x_{i}\right)\right)}{\left(\hat{q}_{\alpha_{2}}\left(x_{i}\right)-\hat{q}_{\alpha_{1}}\left(x_{i}\right)\right)}}{\frac{1}{n} \sum_{i=1}^{n} \tau\left(x_{i}\right) w\left(\hat{q}_{0.5}\left(x_{i}\right)\right)}
$$

where $\tau\left(\mathrm{X}_{\mathrm{i}}\right)$ is a trimming function, whose support, denoted by $\mathrm{X}_{\tau}$, is a compact set which lies strictly in the interior of $\mathrm{X}$. The trimming function serves to eliminate" boundary effects" that arise in nonparametric estimation. We use the superscript (p) to distinguish the estimator of the median function in this stage from that in the first stage.

3) Local Polynomial Estimation at the Point of Interest Letting $\mathrm{x}$ denote the point at which the function $\mu(\cdot)$ is to be estimated at, we combine the local polynomial estimator, with polynomial order $\mathrm{k}$ and bandwidth sequence $h_{3 n}$, of the conditional quantile function at $x$ using quantiles $\alpha 1, \alpha 2$, with the estimator of the unknown disturbance quantiles, to yield the estimator of $\mu(\mathrm{x})$ :

$$
\hat{\mu}(x)=\hat{c}_{2} \hat{q}_{\alpha_{1}}(x)-\hat{c}_{1} \hat{q}_{\alpha_{2}}(x)
$$

\section{Estimating the Distribution of $\epsilon_{i}$}

As mentioned in Section 2, the distribution of the random variable $\boldsymbol{\epsilon}_{\mathbf{i}}$ is identified for all quantiles exceeding $\alpha_{0} \equiv$ $\inf \left\{\alpha: \sup _{\mathrm{x \in X}} \mathrm{q}_{\alpha}(\mathrm{x})>0\right\}$. In this section we consider estimation of these quantiles, and the asymptotic properties of the estimator. Estimating the distribution of $\mathbf{\epsilon}_{\mathbf{i}}$ is of interest for two reasons. First, the econometrician may be interested in estimating the entire model, which would require estimators of $\sigma(x \mathbf{i})$ and the distribution of $\mathbf{\epsilon}_{\mathbf{i}}$ as well as of $\mu(x i)$. Second, the estimator can be used to construct tests of various parametric forms of the distribution of $\boldsymbol{\epsilon}_{\mathbf{i}}$, and the results of these tests could then be used to adopt a (local) likelihood approach to estimating the function $\mu(x i)$.

Before proceeding, we note that the distribution of $\boldsymbol{\epsilon}_{\mathbf{i}}$ is only identified up to scale, and we impose the scale normalization that $\mathrm{c} 0.75-\mathrm{c} 0.25 \equiv 1$. We also assume without loss of marketingity that $\alpha 0 \leq 0.25$. To estimate $\mathrm{c}_{\alpha}$ for any $\alpha \geq \alpha_{0}$, we let $\alpha_{-}=\min (\alpha, 0.5)$ and define our estimator as

$$
\hat{c}_{\alpha}=\frac{\frac{1}{n} \sum_{i=1}^{n} \tau\left(x_{i}\right) w\left(\hat{q}_{\alpha_{-}}\left(x_{i}\right)\right) \cdot\left(\hat{q}_{\alpha}\left(x_{i}\right)-\hat{q}_{0.5}^{(p)}\left(x_{i}\right)\right)}{\frac{1}{n} \sum_{i=1}^{n} \tau\left(x_{i}\right) w\left(\hat{q}_{\alpha_{-}}\left(x_{i}\right)\right) \cdot\left(\hat{q}_{0.75}\left(x_{i}\right)-\hat{q}_{0.25}\left(x_{i}\right)\right)}
$$

The proposed estimator, which involves averaging nonparametric estimators, will converge at the parametric ( $\sqrt{ }$ ) rate and have a limiting normal distribution, as can be rigorously shown using similar arguments found in Chen and Khan(1999b). 


\section{International Journal of Science and Research (IJSR)}

ISSN (Online): 2319-7064

Index Copernicus Value (2013): 6.14 | Impact Factor (2015): 6.391

\section{Monte Carlo Results}

In this section the finite sample properties of the proposed estimator are explored by way of a small scale simulation study. We simulated from designs of the form:

$$
y_{i}=\max \left(\mu\left(x_{i}\right)+\sigma\left(x_{i}\right) \epsilon_{i}, 0\right)
$$

where xi was a random variable distributed uniformly between -1 and $1, \boldsymbol{\epsilon}_{\mathbf{i}}$ was distributed standard normal, and the scale function $\sigma(x i)$ was set to $e^{0.15 x i}$. We considered four different functional forms for $\mu(x i)$ in our study:

$$
\begin{aligned}
& \text { 1. } \mu(x)=x \\
& \text { 2. } \mu(x)=x^{2}-\mathcal{C}_{1} \\
& \text { 3. } \mu(x)=0.5 \cdot x^{3} \\
& \text { 4. } \mu(x)=e^{x}-\mathcal{C}_{2}
\end{aligned}
$$

where the constants $\mathrm{C} 1, \mathrm{C} 2$ were chosen so that the censoring level was $50 \%$, as it was for the other two designs.

We adopted the following data-driven method to select the quantile pair. For a given point $\mathrm{x}$, we note that the estimator requires that $\mathrm{q}_{\alpha 1}(\mathrm{x}), \mathrm{q}_{\alpha 2}(\mathrm{x})$ both be strictly positive for identification, requiring that the quantiles be sufficiently close to 1 . On the other hand, efficiency concerns would suggest that the quantiles not be at the extreme, as the quantile regression estimator becomes imprecise. We thus let the probability of being censored, or the "propensity score" (see Rosenbaum and Rudin(1983)) govern the choice of quantiles for estimating the function $\mu(\cdot)$ at the point $x$. Letting di denote an indicator function which takes the value 1 if an observation is uncensored, we note that;

$$
1-E\left[d_{i} \mid x_{i}=x\right]=F_{\epsilon}\left(\frac{-\mu(x)}{\sigma(x)}\right)
$$

where $F_{\epsilon}(\cdot)$ denotes the c.d.f. of $\epsilon_{i}$. Letting $\alpha^{*}=F_{\epsilon}\left(\frac{-\mu(x)}{\sigma(x)}\right)$, we note that

$$
\begin{aligned}
q_{\alpha^{*}}(x) & =\max \left(\mu(x)+c_{\alpha^{*}} \sigma(x), 0\right) \\
& =\max \left(\mu(x)+\frac{-\mu(x)}{\sigma(x)} \sigma(x), 0\right) \\
& =0
\end{aligned}
$$

Thus if one knew the propensity score value, identification would require that $\alpha^{*}$ be a lower bound for the choice of quantile pair. The propensity score can be easily estimated using kernel methods, suggesting an estimator of $\alpha^{*}$ :

$$
\hat{\alpha}^{*}=1-\frac{\frac{1}{n} \sum_{i=1}^{n} d_{i} K_{h}\left(x_{i}^{(c)}-x^{(c)}\right) I\left[x_{i}^{(d)}=x^{(d)}\right]}{\frac{1}{n} \sum_{i=1}^{n} K_{h}\left(x_{i}^{(c)}-x^{(c)}\right) I\left[x_{i}^{(d)}=x^{(d)}\right]}
$$

where $K_{h}(\cdot)=\hbar^{-d_{c}} K(\dot{\bar{h}})$ where $\hbar$ is a bandwidth sequence, and $K(\cdot)$ is a kernel function.

Our proposed choice of quantile pair takes into account this lower bound as well as the efficiency loss of estimating quantiles at the extreme. We set:

$$
\alpha_{1}=\frac{2 \hat{\alpha}^{*}+1}{3} \quad \alpha_{2}=\frac{2+\hat{\alpha}^{*}}{3}
$$

which divides the interval $\left[\alpha^{*}, 1\right]$ into three equal spaces. In implementing this procedure in the Monte Carlo study, the propensity scores were estimated using a normal kernel function and a bandwidth of $\mathrm{n}^{-1 / 5}$.

For the quantile estimators, a local constant was fit in the first stage, using a bandwidth of $\mathrm{n}^{-1 / 5}$, and a local linear estimator was used in the second and third stages, using a bandwidth of the form $\mathrm{kn}^{-1 / 5}$. The constant $\mathrm{k}$ was selected using the "rule of thumb" approach detailed on page 202 in Fan and Gijbels(1996).

\section{Case Studies}

\subsection{Application Tobit regression to plot employees}

Consider the situation in which we have a measure of management aptitude (scaled 200-800) which we want to model using psycotesting and math test scores, as well as, the type of program the student is enrolled in (management, marketing, or administration). The problem here is that students who answer all questions on the management aptitude test correctly receive a score of 800 , even though it is likely that these students are not "truly" equal in aptitude. The same is true of students who answer all of the questions incorrectly. All such students would have a score of 200 , although they may not all be of equal aptitude.

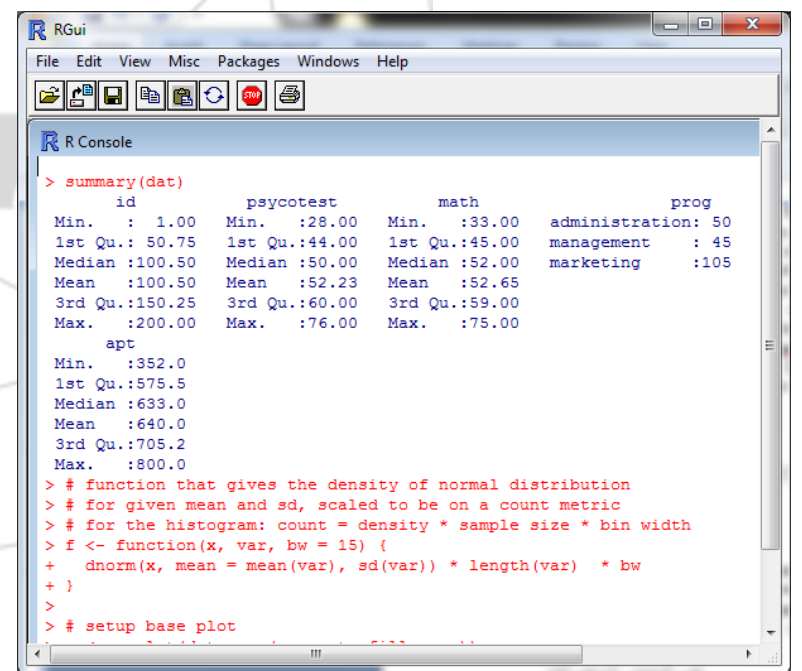


International Journal of Science and Research (IJSR)

ISSN (Online): 2319-7064

Index Copernicus Value (2013): 6.14 | Impact Factor (2015): 6.391

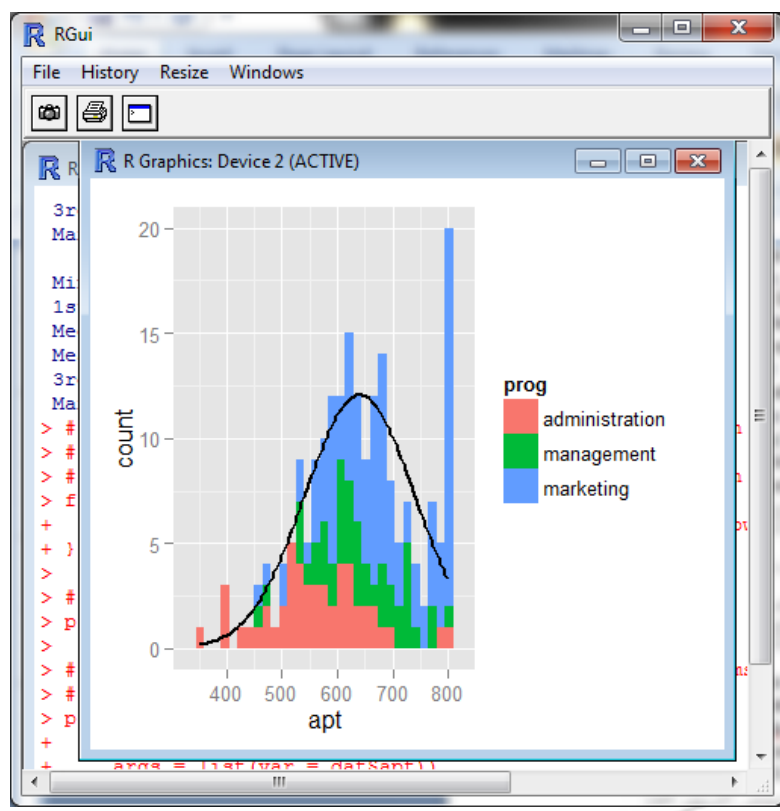

Looking at the above histogram, we can see the censoring in the values of apt, that is, there are far more cases with scores of 750 to 800 than one would expect looking at the rest of the distribution. Below is an alternative histogram that further highlights the excess of cases where $\mathbf{a p t}=800$. In the histogram below, the breaks option produces a histogram where each unique value of apt has its own bar (by setting breaks equal to a vector containing values from the minimum of apt to the maximum of apt). Because apt is continuous, most values of apt are unique in the dataset, although close to the center of the distribution there are a few values of apt that have two or three cases. The spike on the far right of the histogram is the bar for cases where apt $=800$, the height of this bar relative to all the others clearly shows the excess number of cases with this value.

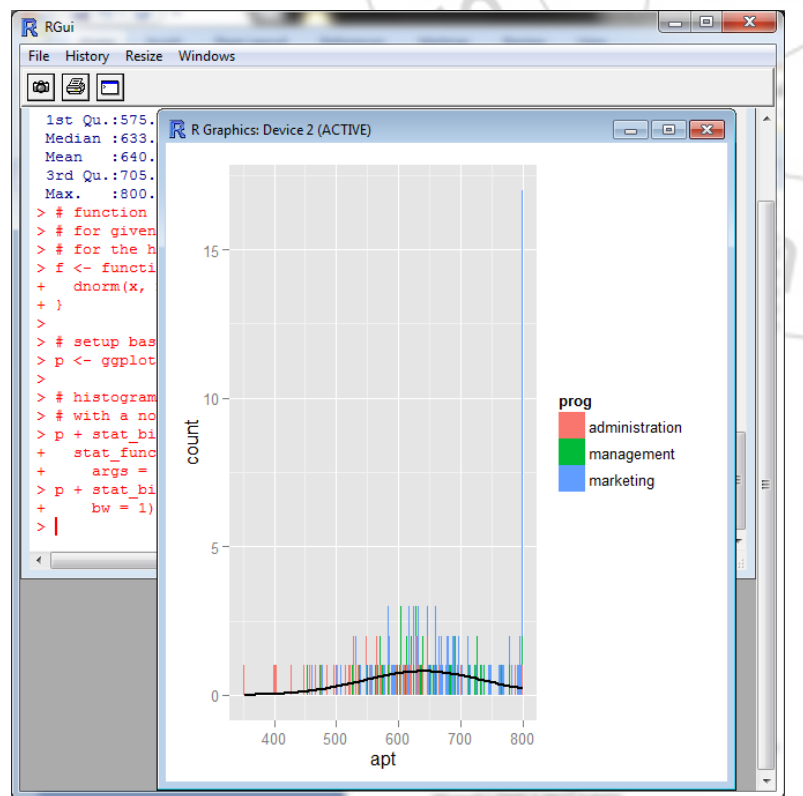

Next we'll explore the bivariate relationships in our dataset.

psycotest math apt

psycotest 1.00000000 .66228010 .6451215 math 0.66228011 .00000000 .7332702

apt 0.64512150 .73327021 .0000000
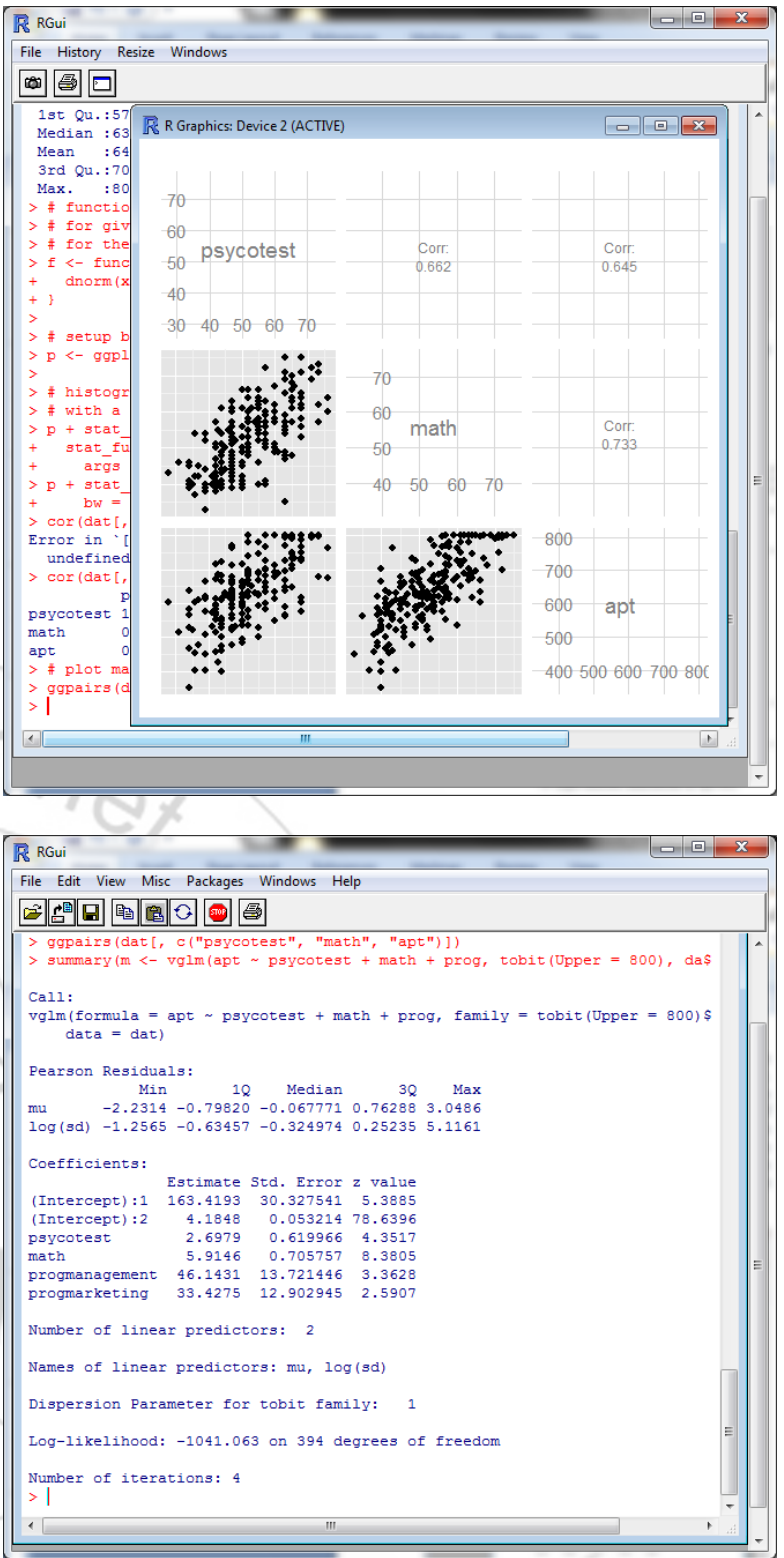

\section{Conclusion}

- In the output above, the first thing we see is the call, this is $\mathrm{R}$ reminding us what the model we ran was, what options we specified, etc.

$\circ$ The table labeled coefficients gives the coefficients, their standard errors, and the z-statistic. No p-values are included in the summary table, but we show how to calculate them below. Tobit regression coefficients are interpreted in the similar manner to OLS regression coefficients; however, the linear effect is on the uncensored latent variable, not the observed outcome.

$\circ$ For a one unit increase in psycotest, there is a $\mathbf{2 . 6 9 8 1}$ point increase in the predicted value of apt.

$\circ \mathrm{A}$ one unit increase in math is associated with a 5.9146 unit increase in the predicted value of apt.

$\circ$ The terms for prog have a slightly different interpretation. The predicted value of apt is $\mathbf{- 4 6 . 1 4 1 9}$ 


\section{International Journal of Science and Research (IJSR) \\ ISSN (Online): 2319-7064}

Index Copernicus Value (2013): 6.14 | Impact Factor (2015): 6.391

points lower for students in a administration program than for students in an management program.

o The coefficient labeled "(Intercept): 1 " is the intercept or constant for the model.

o The coefficient labeled "(Intercept):2" is an ancillary statistic. If we exponentiate this value, we get a statistic that is analogous to the square root of the residual variance in OLS regression. The value of 65.6773 can compared to the standard deviation of management aptitude which was 99.21, a substantial reduction.

- The final log likelihood, -1041.0629 , is shown toward the bottom of the output, it can be used in comparisons of nested models.

- For a one unit increase in psycotest, there is a 2.7 point increase in the predicted value of apt.

- A one unit increase in math is associated with a 5.91 unit increase in the predicted value of apt.

- The terms for prog have a slightly different interpretation. The predicted value of apt is 46.14 points lower for students in a administration program (prog=3) than for students in an management program (prog=1).

The tobit model, also called a censored regression model, is designed to estimate linear relationships between variables when there is either left- or right-censoring in the dependent variable (also known as censoring from below and above, respectively). Censoring from above takes place when cases with a value at or above some threshold, all take on the value of that threshold, so that the true value might be equal to the threshold, but it might also be higher. In the case of censoring from below, values those that fall at or below some threshold are censored.

Interval regression is used to model outcomes that have interval censoring. In other words, you know the ordered category into which each observation falls, but you do not know the exact value of the observation. Interval regression is a marketingization of censored regression.
[6] Chen, S. and S. Khan (2001) "Semiparametric Estimation of a Partially Linear Censored Regression Model", Econometric Theory, 17, 567-590.

[7] Fan, J. (1992) "Design-adaptive Nonparametric Regression", Journal of the American Statistical Association, 87, 998-1004.

[8] Fan, J. and I. Gijbels (1996) Local Polynomial Modelling and its Applications, New York: Chapman and Hall.

[9] Khan, S. (2001) "Two Stage Rank Estimation of Quantile Index Models", Journal of Econometrics, 100, 319-355.

[10] Koenker, R. and G.S. Bassett Jr. (1978) "Regression Quantiles", Econometrica, 46, 33-50.

[11] Powell, J.L. (1984) "Least Absolute Deviations Estimation for the Censored Regression Model", Journal of Econometrics, 25, 303-325.

[12] Rosenbaum, P.R. and D.B. Rubin (1983) "The Central Role of the Propensity Score in Observational Studies for Causal Effects", Biometrica, 70, 41-55

\section{References}

[1] Chaudhuri, P. (1991a) "Nonparametric Quantile Regression", Annals of Statistics, 19, 760-777.

[2] Chaudhuri, P. (1991b) "Global Nonparametric Estimation of Conditional Quantiles and their Derivatives", Journal of Multivariate Analysis, 39, 246-269.

[3] Chaudhuri, P., K. Doksum, and A. Samarov (1997) "On Average Derivative Quantile Regression", Annals of Statistics, 25, 715-744.

[4] Chen, S., Dahl. G. B., and S. Khan (2002) "Nonparametric Identification and Estimation of a Censored Regression Model with an Application to Unemployment Insurance Receipt", Center For Labor Economics University of California, Berkeley Working Paper no.54

[5] Chen, S. and S. Khan (2000) "Estimation of Censored Regression Models in the Presence of Nonparametric Multiplicative Heteroskedasticity", Journal of Econometrics, 98, 283-316. 